\title{
De Novo Modeling in Cryo-EM Density Maps with Pathwalking
}

\author{
Muyuan Chen ${ }^{\mathrm{a}, \mathrm{c}}$, Philip R. Baldwin ${ }^{\mathrm{b}, \mathrm{c}}$, Steven J. Ludtke ${ }^{\mathrm{c}}$, Matthew L. Baker ${ }^{\mathrm{c}}$ \\ ${ }^{a}$ Program in Structural and Computational Biology and Molecular Biophysics \\ ${ }^{b}$ Department of Psychology \\ ${ }^{c}$ Verna and Marrs McLean Department of Biochemistry and Molecular Biology, Baylor College of \\ Medicine, Houston, TX 77030
}

\begin{abstract}
As electron cryo-microscopy(cryo-EM) can now frequently achieve near atomic resolution, accurate interpretation of these density maps in terms of atomistic detail has become paramount in deciphering macromolecular structure and function. However, there are few software tools for modeling protein structure from cryo-EM density maps in this resolution range. Here, we present an extension of our original Pathwalking protocol, which can automatically trace a protein backbone directly from a near-atomic resolution (3 - $6 \AA$ ) density map. The original Pathwalking approach utilized a Traveling Salesman Problem solver for backbone tracing, but manual adjustment was still required during modeling. In the new version, human intervention is minimized and we provide a more robust approach for backbone modeling. This includes iterative secondary structure identification, termini detection and the ability to model multiple subunits without prior segmentation. Overall, the new Pathwalking procedure provides a more complete and robust tool for annotating protein structure function in near-atomic resolution density maps.
\end{abstract}

Keywords: protein structure, backbone model

\section{Introduction}

While cryo-Electron Microscopy(cryo-EM) has traditionally been limited to low resolution [1], it is now possible to produce structures for large macromolecular complexes at better than $3 \AA$ resolution[2] [3] [4] [5], largely due to technological advances in electron detector technology[6]. While such high resolution cryo-EM structures have been achieved in a handful of cases, the vast majority of cryo-EM reconstructions achieve lower resolution, making interpretation much more challenging [7] [8] [9]. At these resolutions, the pitch of helices, separation of individual strands in $\beta$-sheets and even some bulky sidechains may be visible in the density map but often lack the detail for traditional protein structure modeling[10].

As such, the ability to accurately interpret a density map in this resolution range is paramount to understanding structure and function in macromolecules. When available, high resolution structural models from X-ray crystallography and NMR are often fit to a cryo-EM density map in order to produce atomic level information about the complex[11]. 
In some instances, the high resolution structural model is allowed to deform in order to best fit the density map[12]. Computational modeling tools have also been used to generate, score and select models based density constraints[13] [14] [15] [16] [17]. In addition, lower resolution annotations, such as secondary structure localization [18] [19] and domain segmentation[20] [21], can also be used gain insight into macromolecular structure and function despite the lack of atomic detail[22] [23] [24].

While lacking true high resolution detail, many of the cryo-EM density maps between $3-6 \AA$ resolution contain enough information to build robust structural models for the individual components directly from a density map[25] [26] [27] [28] [29] [30] [31] [32] [33]. Under ideal circumstances, the path of the backbone through a density map is unambiguous. That is, a human examining the structure in detail could accurately trace a protein backbone through the density map without any branching, breaking or other ambiguities in the density map. However, in the majority of density maps at near atomic resolution this is not true, as some ambiguities, due to imaging and reconstruction errors and noise, will likely exist. In addressing these issues, we developed a procedure, Pathwalking, to enumerate plausible backbone paths through a density map[34] [35].

Pathwaking is based on the Traveling Salesman Problem (TSP), in which possible cyclical paths (the protein fold) are calculated through a density map without using any sequence or structure constraints[36] [37]. Essentially, the only required input data for Pathwalking is a segmented density map better than $6 \AA$ resolution and the number of amino acids in the protein. Briefly, the protocol starts with secondary structure identification in the density map using software such as SSEHunter[38]. Pseudoatoms are then generated directly in the density map, representing the $\mathrm{C} \alpha$ atoms in the protein to be modeled. In the next step, the shortest path that visits each pseudoatom only once is assessed using the TSP solver. Essentially, the TSP solver finds the path through the pseudoatoms by optimizing the spatial distance between the pseudoatoms such that they are representative of $\mathrm{C} \alpha$-C $\alpha$ distances in consecutive amino acids in the protein structure. The path is then evaluated manually and the position of pseudoatoms adjusted. A new path is then calculated based on the updated position of pseudoatoms; this process is often iterated multiple times until an acceptable model is returned.

This approach has produced in a number of relatively accurate structural models from density maps at near-atomic resolutions[39] [26]. However, the Pathwalking procedure was not fully automated, and requires human input at specific stages to guide the overall process. Building on the original Pathwalking approach and motivated by the rapidly growing number of near atomic resolution structures, we present here an enhanced version of our original utilities capable of producing more accurate models with reduced user interaction. In addition to improved model building, Pathwalking now incorporates a secondary structure element (SSE) detection routine into the model building process that further improves the accuracy at which a protein fold is defined.

\section{Methods}

While the original implementation of the Pathwalking protocol was far better than purely manual model building, it did require a considerable amount of human interaction. This means the ultimate accuracy of the model is highly dependent on the skill of the person doing the modeling. The main interactive steps in the original version of Pathwalking includes identifying secondary structures using the SSEHunter[38], setting 
a threshold for the density map before pseudoatom placement, and finally, evaluating the identified paths and setting constraints. Even with the semi-automated approach of Pathwalking, these manual steps could be relatively time-consuming for large proteins. This is further complicated by the fact that multiple models may be required for large structures, making accurate map segmentation a critical issue. In the new version of Pathwalking, we have addressed these problems by introducing additional quantitative assessments and automation. A fully automated modeling process can be performed using the command:

e2pathwalker_auto.py density_map.mrc --natoms=N - -denthr=T

Here $\mathrm{N}$ is the number of residues in the protein and $\mathrm{T}$ is the threshold of the isosurface value in the density map. The program will calculate a default threshold value if --denthr=T is not provided, but a carefully selected threshold value that separates features is still recommended especially at lower resolution. This script will run the programs in our new Pathwalking workflow using the default parameters, and generate the output for each step.

It should be noted that the single e2pathwalker executable does not assign the sequence to the trace, rather the it is simply an enumerated poly-alanine C-alplha backbone trace. The EMAN2 companion program, e2seq2pdb.py, will do both a forward and reverse sequence assignment to the trace. It is also important to note that, like the original version of Pathwalking, no explicit sequence information is utilized. As such, no registration of strucural details such as SSEs or bulky sidechains are used in modeling. However, this type of information can be used to independently validate models generated from Pathwalking.

While the fully automated protocol can generate decent models when the input density map is well resolved, users may also opt to run each program in the protocol step by step to adjust parameters and access advanced options. This is particularly useful when attempting to apply additional structural constraints. All output models are in standard pdb format so users can view the results in molecular visualization software, such as UCSF's Chimera [40]. A detailed tutorial can be found online at http://blake.bcm.edu/emanwiki/Pathwalker. In the following sections we detail the algorithmic and automation improvements in each step of our new protocol (Fig. 1).

\subsection{Pseudoatom placement}

In the first step of the Pathwalking protocol(Fig. 1B), the density map is first mapped onto a Cartesian grid with a grid spacing of $3.8 \AA$, the average distance between $\mathrm{C} \alpha$ atoms in peptides. While there is no reason to expect that we are going to identify precise $\mathrm{C} \alpha$ locations rather than random locations along the protein fold, this scale is representative of the level at which we need to characterize the structure. N pseudoatoms are first placed in the center of cubes with the $\mathrm{N}$ highest mean densities, where $\mathrm{N}$ is the number of amino acids in the protein. K-means clustering is then performed using these points as initial cluster centers and all the points above a certain threshold in the density as samples. The threshold is set to be the lowest mean density of the $\mathrm{N}$ grid points by default. The k-means method has been modified so that the new center positions in each iteration are a density weighted average of the member points rather than an unweighted average. This pseudoatom seeding process terminates when the relative motion of cluster centers falls below a small threshold value. 


\subsection{Path detection}

After pseudoatom generation, path detection is performed using a TSP solver(Fig. 1C). Pathwalking is compatible with both the Concorde [36] and LKH [37] TSP solvers. Here, the paths are evaluated by biochemical constraints and the fitness to the density map. A distance matrix is created in which the distance between two pseudoatoms is expressed in terms of the cost function to be an actual $\mathrm{C} \alpha-\mathrm{C} \alpha$ "bond". This cost function is calculated using the distance between the two atoms expressed as a deviation from the optimal $3.8 \AA \mathrm{C} \alpha-\mathrm{C} \alpha$ distance and the density value between the atoms.

$$
\left\{\begin{array}{l}
(d-3.78)^{2}, d<m a x \_d i s t, \\
(d-3.78)^{4}, d \geq m a x_{\_} d i s t
\end{array}+w *\left(1 / a v g_{-} d e n\right) *\left\{\begin{array}{l}
2, a v g_{-} d e n>d e n_{-} t h r \\
1, \text { avg_den }<=d e n_{-} t h r
\end{array}\right.\right.
$$

Here $d$ is the distance between the two pseudoatoms, and avg_den is the average density of all voxels on a straight line between them. max_dist and den_thr are the maximum distance and density threshold that can be set by the user. This distance matrix is then used as the input for the TSP solver, which attempts to find the lowest cost path of the protein through the density map.

\subsection{Path refinement}

A particularly complicated problem for Pathwalking is the fact that different features in the map may have significantly different relative density values. If a single density threshold is used, the path can be either disconnected or highly ambiguous. This is particularly evident with SSEs at near atomic resolution; at higher thresholds helices are typically well resolved but coils and strands are usually not discernible. When lowering the density threshold, SSEs often merge together, again making it hard for accurately discriminate density features.

To overcome these issues, we implemented an iterative path refinement step based on automatic SSE identification. Secondary structure elements are firstly identified based on the initial path from Pathwalking as well as geometric criteria which are further detailed in the following sections(Fig. 1D). Pseudoatoms in these secondary structures are then fixed to their ideal positions. After this, the density corresponding to these SSEs are removed from the density map and the remaining pseudoatoms are seeded again on the modified density map(Fig. 1E). A new path is then computed based on these pseudoatoms, including those "locked" from the previous round of secondary structure elements identification. By repeating this process, more and more secondary structure elements are identified, in addition to refining both pseudoatom placement and path identification. Model refinement stops when there are no more SSEs found in an iteration(Fig. 1F)

\subsubsection{Secondary Structure Identification: $\alpha$-helices}

After an initial path is generated, SSE detection first identifies long helical/cylindrical structures along the path. Here, helical shape is defined as a subpart of the path that has all its pseudoatoms within a range of distances to the central axis of these points. For each pseudoatom, we sum the number of pseudoatoms extending in both directions that maintains an $\alpha$-helical profile, and set this value as the helical score of this pseudoatom(Fig. 5C). Potential helices are identified using this score. 
To confirm these putative helices, the algorithm generates ideal pseudoatoms for a helical path at these sites and fits them to the density map; potential helices can be identified by the fitness of ideal helices. For the confirmed helices, pseudoatoms in the helices are replaced by the ideal $\alpha$ helices. The length and position of these helices are then optimized based on the density map.

After the helix fitting step, a density map with helical density removed is generated. The program then fix the pseudoatoms on helices and re-seeds the rest pseudoatoms. The path tracing and helix detection is repeated until no new helices are found.

\subsubsection{Secondary Structure Identification: $\beta$-sheets}

While helix identification can generally be performed at resolutions as low as $6-8 \AA$ in cryo-EM density maps, $\beta$-sheet tracing is typically best suited for maps at better than $4.5 \AA$ resolution, where the strands in the sheet begins to resolve. For sheet identification, we calculate a score for each pseudoatom indicating the cost function of it belonging to a $\beta$ sheet(Eq. 2). The score is calculated based on the angle between the bonds connecting the pseudoatom and the other bonds near the pseudoatom in the 3D space, so that the score for the pseudoatom is higher when it is in a region where the bonds tend to be parallel to each other, suggesting a potential $\beta$ sheet(Fig. 5A).

$$
\sum_{(k \text { nearest atoms })} P D F_{\text {Gaussian }}(\text { distance, sigma }) * a b s(\cos (\text { angle between bonds }))
$$

Then, the score is smoothed along the backbone chain using a Gaussian moving average, and the algorithm searches for the longest continuous chain in which the pseudoatoms have a score higher than a threshold (mean+std by default). Once a chain has been identified, it is elongated from both ends based on the position of nearby pseudoatoms. After that, the score for all the rest of the pseudoatoms is calculated again while the bonds near and parallel to the existing chains are weighted higher. The process is performed iteratively until the score or length of the next new chain is below a certain threshold.

\subsection{Path modification}

After the refinement completes, the program searches the final path for "crossover bonds", points along the path that should be continuous but exhibit a marked departure in their current direction and forming non-protein-like structure. In most cases, these crossover bonds can be fixed by simply switching the order of residues in the backbone path. If the reordered path still does not meet the geometry criteria, the related pseudoatoms can be randomly shaken in the local region to obtain a better path. In addition to these non-protein like features, pseudoatoms can be placed in large sidechain densities in the pseudoatom generation step. These pseudoatoms can be identified by the density around the atom, length and angle of bonds and the relationship with the main chain. These sidechain atoms are then eliminated from the backbone model. Along with the aforementioned Pathwalking utilities, we have provided a supplemental script for common structural modification in $\mathrm{C} \alpha$ model. In this step, users should evaluate their model and refer to the program documentation regarding these types of modification steps. 


\subsection{Termini determination}

In addition to iterative path improvement, a new step has been incorporated into Pathwalking to better localize the backbone termini. In most instances, the position of terminal pseudoatoms in the density map is unknown before modeling, which can also be complicated by the fact that in many cases the $\mathrm{C}$ and $\mathrm{N}$ termini are near each other in 3-D. The new algorithm identifies the most likely termini based on the geometry of the pseudoatoms. Two phantom points are initially placed in the model as start and end points, and the distance to each pseudoatoms is set to 0. Since the TSP solver is designed to find the path with the minimum total length, it will connect a pseudoatom to this phantom termini when it is the most unlikely to be connected to any other atoms. Finally, the program removes the phantom points and assigns the pseudoatoms directly connecting them as the termini. Although the program can detect the termini automatically, the direction of the path is still ambiguous, which can be resolved by the user using sequence and secondary structure information.

As an added feature, this approach may also be used to model multiple subunits in the same density map that cannot be properly separated/segmented. Again, an extra phantom point is added for each subunit, where adding the phantom point is equivalent to allowing a gap in the calculated path. The TSP solver will find the most separable bond to put the gap in order to minimize the global distance. As such, adding phantom points in the seeding step allows for the tracing additional subunits. In addition, this technique can also be used to build models on density maps with poorly resolved regions. Users can treat a poorly resolved density map as multiple subunit so the program can automatically avoid density breaks/gaps, and build the backbone for only the well resolved parts of the map.

\section{Results}

To evaluate the performance of the Pathwalking protocol, we ran the new, enhanced version of Pathwalking on the real cryo-EM data sets used in the original Pathwalking paper[35], as well as recent near-atomic resolution density maps. For these data, each map also contains a corresponding X-ray crystal structure available from the PDB.

Unlike the previous Pathwalking tests, all of the new tests were done without human intervention, except for fine tuning parameters as noted. The new Pathwalking process is iterative, and all of the models converged within three rounds of refinement. The entire modeling process for a single protein subunit generally took no more than 10 minutes, plus the time spent to compare and assess the model afterwards.

As in the previous paper, the Pathwalking models were evaluated using the CLICK web server[41].The CLICK score is used instead of other, more standard modeling metrics as it compares the model based on the overall fold, is less effected by variations in model path and does not require a sequence alignment. The Pathwalking structures are aligned to the corresponding crystal structure by CLICK. Structural overlap score reported in Table 1 represents the percentage of atoms that are correctly registered after the alignment which describes how good the alignment is. Calculated on the correctly registered atoms, RMSD shows the precision of pseudoatom position and topology score measures the similarity of the path.

We tested the new Pathwalking method on 40 simulated density maps at $5 \AA$ resolution and have achieved a similar or better topology score over a larger region of overlap 
Table 1: Pathwalking results

\begin{tabular}{l|c|c|c|c|c|c|c}
\hline PDB ID & $\begin{array}{c}\text { EMDB } \\
\text { ID }\end{array}$ & $\begin{array}{c}\text { Resolution } \\
(\AA)\end{array}$ & $\begin{array}{c}\text { Number of } \\
\text { Residues }\end{array}$ & $\begin{array}{c}\text { Structure } \\
\text { Overlap**(\%) }\end{array}$ & $\begin{array}{c}\text { RMSD** } \\
\text { Topology } \\
\text { Score** }\end{array}$ & $\begin{array}{c}\text { TopSearch } \\
\text { Rank }\end{array}$ \\
\hline 1SS8 & 5001 & 4.2 & 525 & $48.48(10.3)$ & $2.70(7.51)$ & $0.75(0.79)$ & 1 \\
1IH5 & N/A*** & - & 223 & $85.38(27.3)$ & $2.32(4.63)$ & $0.73(1.00)$ \\
1QHD & 1461 & 3.8 & 394 & $82.78(40.8)$ & $2.24(3.99)$ & $0.97(0.93)$ \\
3C5B & 5003 & 4.5 & 334 & $52.84(5.1)$ & $2.68(9.02)$ & $0.89(0.75)$ \\
3J7L & 6000 & 3.8 & 164 & 79.88 & 2.05 & 1.00 & 1 \\
3J4U:N & 5764 & 3.5 & 140 & 81.43 & 2.06 & 1 \\
3J4U:C & 5764 & 3.5 & 327 & 72.78 & 2.45 & 0.79 \\
3J5P & 5778 & 3.28 & 592 & 10.74 & 2.69 & 1.00 \\
3J6B:C & 2566 & 3.2 & 249 & 92.77 & 1.76 & 1.00 \\
3J6B:I & 2566 & 3.2 & 125 & 98.90 & 2.12 & 1 \\
3J6B:M & 2566 & 3.2 & 151 & 92.97 & 1.94 & 1 \\
1Z56:C & $\mathrm{N} / \mathrm{A} * * *$ & - & 237 & 61.60 & 2.61 & 1 \\
\hline
\end{tabular}

* The entries in the list are GroEL chain A(1SS8), Aquaporin(1IH5), Rotavirus VP6(1QHD), Epsilon15 GP7(3C5B), Brome mosaic virus(3J7L), Bacteriophage BPP-1(3J4U), TRPV1(3J5P), Yeast mitochondrial large ribosomal subunit(3J6B), DNA ligase IV chain C(1Z56).

** Previously reported scores from the original Pathwalking assessment are shown in parenthesis.

*** Maps from X-ray crystallographic data

than the previous protocol (Supplement Table 1). Additionally, the new Pathwalking routines were tested on four authentic cryo-EM density maps tested from the original paper[42][43][44][45] and achieved similar topology scores, as well as a higher proportion of correctly registered $\mathrm{C} \alpha$ and lower RMSD, when compared to the previous version(Fig. 2, Table 1). This indicates that the new version of Pathwalking can automatically build models as good or better than the previous human-guided method. Specifically, the relatively high topology scores show that the new Pathwalking approach can generate highfidelity first approach models that are equivalent to their known structure. Moreover, we searched the PDB database for the structures most similar to the results generated by Pathwalker using TopSearch[46]. In all cases listed in the table, the model of the target protein or its homolog is listed at the first or second rank, suggesting that our protocol captures the general fold of the protein. The improved RMS deviation also suggests that the modified pseudoatom seeding strategy and path correction can improve the overall quality of the model. We also tested the SSE identification protocol on simulated density map. Additionally, we tested and showed that new protocol can identify most of the SSEs accurately; successful SSE identification can improve the CLICK score of the model in the next iteration. (Supplement Table 2,3)

While all of the models report high topology scores, a closer investigation into the TRPV1 dataset (Fig. 2G, Table 1) reveals that the model generated by Pathwalking has a different fold when compared to the known model (PDB ID: 3J5P)[47][48]. This is mainly attributed to the missing density in the ankyrin repeat domain at the N-terminus and additional unmodeled density near the $\beta$ sheets in the C-terminus. To model the additional density and maintain the connectivity of the model, the algorithm chose an alternative path, connecting the C-terminus to the linker domain instead of the TRP 
domain, thus reversing the chain direction in the transmembrane core. Although the resulting model is still visually similar to the reference, the CLICK web server is unable to align the two models due to the large scale of the chain reverse; only $10 \%$ of the $\mathrm{C} \alpha$ atoms are correctly registered. CLICK still gives a topology score of 1 , which is unlikely to be meaningful given the poor alignment.

To test the new Pathwalking at different resolutions, we ran it on the density maps of Brome mosaic virus(BMV) generated from different subset of raw images (Fig. 4, Table 2)[49]. In this test, density maps reconstructed from $1 / 2,1 / 4,1 / 8,1 / 16$ of the original dataset are used, which corresponded to resolutions from 4.1 to $5.4 \AA$. The models generated from the first three density maps are almost identical, and are topological equivalent to the existing model, while one pair of misconnection occurred in the $5.37 \AA$ resolution dataset. It should be noted that the BMV capsid protein is $\beta$-sheet rich and, at $5.37 \AA$ resolution, separation of the strands can not be seen. Despite the lack of resolvability, Pathwalking is still able to accurately place and connect nearly $2 / 3$ of the pseudoatoms in this map with $2.5 \AA$ RMS deviation.

In addition to assessing effects of resolution on Pathwalking, we explored its ability to model multiple subunits in a density map (Fig. 3, Table 1). Here, we used the new Pathwalking tools on a yeast mitochondrial large ribosomal subunit density map at $3.2 \AA$ resolution containing three adjacent subunits[50]. An initial segmentation of the density corresponding to all three subunits was done using UCSF Chimera[40]. However, no additional segmentation was done, leaving a single density map with the equivalent of the three chains. Without any further localization of the three subunits in the density map or identifying the termini, the new Pathwalking approach was able to segment the density correctly and generate three backbone models for the three ribosome chains. In the three chains, chain $\mathrm{C}$ and $\mathrm{M}$ are topologically identical to the ground truth model, while chain I had some differences due to an obvious gap in the density map, as well as a corresponding one in the reference model, which was chosen as one terminus by the algorithm.

Table 2: Pathwalking performance in different resolutions

\begin{tabular}{l|c|c|c|c}
\hline Fraction of particles used & Resolution & Structure Overlap & RMSD & Topology Score \\
\hline 1 & 3.8 & 79.88 & 2.05 & 1.00 \\
$1 / 2$ & 4.1 & 83.22 & 2.15 & 1.00 \\
$1 / 4$ & 4.2 & 81.88 & 2.19 & 1.00 \\
$1 / 8$ & 4.3 & 83.89 & 1.90 & 1.00 \\
$1 / 16$ & 5.37 & 67.11 & 2.53 & 0.82 \\
\hline
\end{tabular}

In addition to the cryo-EM density maps, we also applied the new Pathwalking protocol on a X-ray crystallography density map. In the case of Xrcc4-DNA ligase IV, which was solved to $3.92 \AA$ resolution [51], our program produced a backbone model that is almost identical to the existing model in the subunit $\mathrm{C}$ (Fig. 2H, Table 1). This result suggests that Pathwalking may be a useful tool for modeling protein structure at near atomic resolution irrespective of the imaging modality, provided the X-ray phases are sufficiently accurate at sufficient resolution. Certainly there will be cases where phasing errors induce density changes which the backbone tracing to change. This, in fact, is the primary reason why CryoEM maps are often perceived as being better than crystal 
structures at the same resolution. CryoEM maps have overall accurate phases, and the resolution limiting factor is simply residual noise, which is far less likely to induce tracing errors.

\section{Discussion}

The new version of our Pathwalking software brings full automation to the protein model building process at near atomic resolution. In most cases, the program can automatically produce a result as good as the result of the original version of Pathwalker without any potential user bias. With an ever increasing number of complex macromolecular structures solved at non-atomic resolutions, Pathwalking now offers a remarkably robust and accurate model building solution that is both easy to use and reliable, even with the most complicated of density maps.

In the original version of Pathwalking, the major manual steps include adjusting atom positions and fixing bond connections in each iteration. In this improved method, the modified seeding strategy can provide better pseudoatom position than human modeler in most cases. The on-grid seeding method gives a better starting position for the $\mathrm{k}$ means clustering, thus can avoid seeding on "dust", pieces of un-attached density, and small sidechains. Additionally, the incorporation of automated routines that replicate and replace those performed manually help to increase the reproducibility and reliability of the modeling process.

Perhaps the biggest advancement of the new routine, the Pathwalking utilities now use the average density value between atoms in the distance matrix for calculating the the shortest path with the TSP solver. Originally, the density map was used merely to seed the pseudoatom positions. The incorporation of the density allows us to achieve more accurate bond connectivity, greatly reducing the incorrect bond connections from nonoptimized pseudoatom position, and largely preventing the connection of a "sidechain pseudoatom" to its neighbor in the chain. This density constraint also helps to avoid the "zig-zag" patterns in the $\beta$-sheet regions that were common, but incorrect, in the original version. While the utilization of the density constraint is automated, the weight of the distance and density value between atoms can be adjusted in certain cases based on map resolvability or to build alternative paths. Generally, at higher resolution, the path is not sensitive to the weight parameter. When the density is more ambiguous and less well resolved, a lower weight should be placed on the map density value so the path will focus more on the pseudoatoms geometry.

Another major accomplishment in the new version of Pathwalking is the incorporation of SSE detection directly into the program. Previously, users could provide the the position of potential helices and $\beta$ sheets from SSEHunter[38], though, it was not directly coupled to Pathwalking. As such, manual intervention was required to incorporate these elements into the Pathwalking protocol as well as evaluate the model in the context of these predictions. To avoid this step, the new version of Pathwalker provides an automated secondary structure identification method performed after each backbone tracing step. The main advantage of this method is that, in addition to the density map, it also makes use of the information encoded by the pseudoatom position and initial backbone contour. In particular, the initial path trace can provide coarse level detail on the location of secondary structure elements in the density map. In turn, this information can then be used to improve backbone tracing in subsequent iterations of 
the Pathwalking protocol. Empirically, SSE identification appears to converge within three iterations with the most significant improvements in SSE geometry coming in the first refinement cycle. Subsequent iterations tend to focus on fine-tuning the placement of pseudoatoms within the density and improvements near the termini of the SSEs. In examining the localization of SSEs by Pathwalking, the results indicate that most straight helices longer than two turns and planar beta-sheets with at least three strands can be accurately identified. Similar SSE localization results are returned with our exhaustive geometrical search routine, SSEHunter. It should be noted though, that like SSEHunter, no explicit directionality is conferred during the SSE localization process. Mapping of the sequence and comparison of map and model features (i.e. SSEs, bulky sidechains) is necessary to define the explicit direction of the path.

As an extension of seeding pseudoatom placement with SSEs, the new Pathwalking protocol can also use known structures to seed pseudoatom placement. In essence, structure fragments or partial models can anchor model building by Pathwalking. This is done simply by inputting the known part of model at the pseudoatom seeding step so that the program will use the "known model" as the optimized model from a previous iteration, then seed the rest of the pseudoatoms before tracing the path. This function can be particularly useful while modeling from a known homolog.

In the previous version, one single subunit of the target protein had to be first segmented out of the full density map before building a model. Additionally, the terminal residues had to be manually identified. In the improved Pathwalking, we show that the program can trace the backbone of multi-subunit complexes simultaneously without the input of terminal residues. While modeling single protein subunits, the algorithm can automatically assign the correct terminus points in most cases, and only fails when there are other obvious gaps in the density map. This function can also be helpful in modeling density maps where the segmentation is poor or ambiguous.

Despite its performance on the test datasets, the improved Pathwalking still has several limitations. The major factor in model accuracy is map resolution. As shown in the result of BMV dataset, the algorithm starts to make minor errors when the resolution falls past $5 \AA$, as the lack of strand separation beyond $5 \AA$ limits the interpretation of $\beta$-strands. However, even in lower resolution maps, the program can still generate a

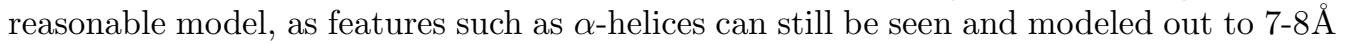
resolution [52]. While certainly not of the same quality as higher resolution models, these models based on low resolution maps maps are still able to provide a general structural overview that can guide new research.

Another potential limitation in Pathwalking can be attributed to the completeness of density map. As our algorithm mainly relies on the density map as input, any missing or additional density in the density map may result in partially correct models.

Small amounts of disordered density, particularly near the termini, usually do not impact the overall model structure, rather small register shifts are introduced throughout the trace. However, large portions of missing density, superfluous density or poorly resolved density regions will have a significant impact on the model. For example, in the case of TRPV1, the additional unmodeled density near the C-terminus causes the program to reverse the chain direction in the transmembrane core. In some instances, such issues can be solved by allowing for several gaps in the path, but it may still need manual validation and correction.

As the core of this method is framed around the, NP-hard, traveling salesman prob- 
lem, the algorithm may fail to converge to an optimal solution in the cases of large complexes with the currently available computational power. The additional modifications made in this version also place additional constraints, making it harder and take more time to converge to the optimal solution. In all reported structures above, the path tracing process takes than 1 minute on one core on a modern desktop computer. Based on our testing, the method may fail to give the optimal solution when there are more than 1500-2000 residues in the complex. For large complexes, segmentation is still recommended before modeling.

In practice, the Pathwalking run into three type of failures. First, the path may appear to be not smooth. Sharp spikes may be observed on the backbone, and the path may cross itself in some local regions. This usually occurs in high resolution structures, while some of the pseudoatoms are seeded on the bulky sidechains. This can be solved by low-pass filtering the density map and running the path modification program provided with Pathwalker, or simply remove the pseudoatoms on the sidechains manually.

The second type of failure is large scale geometry error. In this case, users can see very long bond or other non-protein like pseudoatom connections. Also, very high cost score can be seen on some bonds in the Pathwalking output. This is usually very obvious because a maximum cap is set on the cost function between two pseudoatoms and the TSP solver prefers placing the error on a few connections instead of spreading the error among many pseudoatoms. This type of failure is generally caused by missing density in some part of the protein. This type of problem can be solved by treating the density map as a multi-subunit complex. By setting a number of subunits in the command, the program can break the path into multiple fragment, and the two ends of the missing density are treated as subunit termini. However in this case, there is no information to discriminate between density gap and real termini of the protein, so the user has to evaluate the path manually and make the decision with external knowledge.

The last type of failure during the Pathwalking process is undetected SSEs. Short helices are often missed in the SSE identification, and bent helices are sometimes unidentified or identified as multiple fragments. In either case, the path can remain a rod-like shape and the general fold of rest part of the protein are not affected. $\beta$-sheets are difficult to detect when the resolution is lower and the stands are not separated. The path on the $\beta$-sheets may be misconnected, and sometimes causes incorrect fold in the rest part of the protein. Unfortunately, no automatic solution for this type of problem exists in the current version of Pathwalker; user intervention is required in these cases. Once the connections on the sheets are determined, user can write the connected pseudoatoms in a text file and feed to the Pathwaling program, so the program will optimize the connectivity on the rest part of protein.

Of particular importance in this work is the evaluation of the resulting models from Pathwalking. As mentioned, Pathwalking produces $\mathrm{C} \alpha$ backbone models completely agnostic of the sequence; only a density map and a user provided number for the $\mathrm{C} \alpha$ atoms in the density map are required. The resulting models are essentially non-directional and not assigned any sequence information, i.e. they are represented as a poly-alanine trace. Furthermore, the nodes, or $\mathrm{C} \alpha$ atoms, in the Pathwalking models are representative of the density, and, as such, placement of the $\mathrm{C} \alpha$ atoms is governed by the density and not idealized stereochemistry. Taken together, this means that the models from Pathwalking are essentially initial traces of the protein backbone meant to bootstrap the model building process. Consequently, the initial Pathwalking models may contain 
deviations from the known/ground truth structures. Given this, standard techniques for comparing model accuracy, such as TMscore or GDT, are not optimal for assessing Pathwalking. Rather, techniques like CLICK can report a topology score independent of a sequence alignment, as well as being tolerant of variations in $\mathrm{C} \alpha$ placements. As such, a relative accurate assessment of Pathwalking model topology can be made based solely on the path of the model.

Overall, the new version of Pathwalking, which is available as part of the EMAN2.1 image processing suite[53], represents a significant improvement over the original version, offering both improved performance and new functionality. With the rapidly growing number of structures determined at near-atomic resolutions. Pathwalking provides a unique, robust and reliable way of building models directly from the density map with limited user-based constraints.

\section{Acknowledgements}

This work was supported by grants from the National Institutes of Health (R21GM100229, R01GM079429, P41GM103832 and R01GM080139) and National Science Foundation (DBI-1408887).

\section{References}

[1] Yifan Cheng. Single-Particle Cryo-EM at Crystallographic Resolution. Cell, 161(3):450-457, 2015

[2] Niels Fischer, Piotr Neumann, Andrey L. Konevega, Lars V. Bock, Ralf Ficner, Marina V. Rodnina, and Holger Stark. Structure of the E. coli ribosomeEF-Tu complex at ¡3 Åresolution by Cs-corrected cryo-EM. Nature, 2015.

[3] Melody G Campbell, David Veesler, Anchi Cheng, Clinton S Potter, and Bridget Carragher. 2.8 Åresolution reconstruction of the Thermoplasma acidophilum 20S proteasome using cryo-electron microscopy. eLife, 4, 2015

[4] A. Bartesaghi, A. Merk, S. Banerjee, D. Matthies, X. Wu, J. L. S. Milne, and S. Subramaniam. 2.2 A resolution cryo-EM structure of -galactosidase in complex with a cell-permeant inhibitor. Science, 2015 .

[5] Jiansen Jiang, Bradley L. Pentelute, R. John Collier, and Z. Hong Zhou. Atomic structure of anthrax protective antigen pore elucidates toxin translocation. Nature, 2015.

[6] Xueming Li, Paul Mooney, Shawn Zheng, Christopher R Booth, Michael B Braunfeld, Sander Gubbens, David A Agard, and Yifan Cheng. Electron counting and beam-induced motion correction enable near-atomic-resolution single-particle cryo-EM. Nature methods, 10(6):584-90, 2013.

[7] Juan Esquivel-Rodríguez and Daisuke Kihara. Computational methods for constructing protein structure models from 3D electron microscopy maps. Journal of Structural Biology, 184(1):93-102, 2013.

[8] Matthew L. Baker, Mariah R. Baker, Corey F. Hryc, and Frank DiMaio. Analyses of Subnanometer Resolution Cryo-EM Density Maps, volume 483. 2010.

[9] Wah Chiu, Matthew L. Baker, Wen Jiang, Matthew Dougherty, and Michael F. Schmid. Electron cryomicroscopy of biological machines at subnanometer resolution, 2005.

[10] Matthew L Baker, Junjie Zhang, Steven J Ludtke, and Wah Chiu. Cryo-EM of macromolecular assemblies at near-atomic resolution. Nature Protocols, 5(10):1697-1708, 2010.

[11] Michael G. Rossmann, Marc C. Morais, Petr G. Leiman, and Wei Zhang. Combining X-ray crystallography and electron microscopy, 2005.

[12] Elizabeth Villa and Keren Lasker. Finding the right fit: Chiseling structures out of cryo-electron microscopy maps, 2014.

[13] Frank DiMaio, Michael D. Tyka, Matthew L. Baker, Wah Chiu, and David Baker. Refinement of Protein Structures into Low-Resolution Density Maps Using Rosetta. Journal of Molecular Biology, 392(1):181-190, 2009. 
[14] Maya Topf, Matthew L. Baker, Bino John, Wah Chiu, and Andrej Sali. Structural characterization of components of protein assemblies by comparative modeling and electron cryo-microscopy. Journal of Structural Biology, 149(2):191-203, 2005.

[15] Matthew L. Baker, Wen Jiang, Brian R. Bowman, Z. Hong Zhou, Florante A. Quiocho, Frazer J. Rixon, and Wah Chiu. Architecture of the herpes simplex virus major capsid protein derived from structural bioinformatics. Journal of Molecular Biology, 331(2):447-456, 2003.

[16] Maya Topf, Matthew L. Baker, Marc A. Marti-Renom, Wah Chiu, and Andrej Sali. Refinement of protein structures by iterative comparative modeling and cryoEM density fitting. Journal of Molecular Biology, 357(5):1655-1668, 2006.

[17] Jiang Zhu, Lingpeng Cheng, Qin Fang, Z. Hong Zhou, and Barry Honig. Building and Refining Protein Models within Cryo-electron Microscopy Density Maps Based on Homology Modeling and Multiscale Structure Refinement. Journal of Molecular Biology, 397(3):835-851, 2010.

[18] Matthew L. Baker, Tao Ju, and Wah Chiu. Identification of Secondary Structure Elements in Intermediate-Resolution Density Maps. Structure, 15(1):7-19, 2007.

[19] W Jiang, M L Baker, S J Ludtke, and W Chiu. Bridging the information gap: computational tools for intermediate resolution structure interpretation. Journal of molecular biology, 308(5):1033-1044, 2001.

[20] Matthew L. Baker, Zeyun Yu, Wah Chiu, and Chandrajit Bajaj. Automated segmentation of molecular subunits in electron cryomicroscopy density maps. Journal of Structural Biology, 156(3):432441, 2006.

[21] Grigore Pintilie and Wah Chiu. Comparison of Segger and other methods for segmentation and rigid-body docking of molecular components in Cryo-EM density maps. In Biopolymers, volume 97, pages $742-760,2012$.

[22] Z H Zhou, M L Baker, W Jiang, M Dougherty, J Jakana, G Dong, G Lu, and W Chiu. Electron cryomicroscopy and bioinformatics suggest protein fold models for rice dwarf virus. Nature structural biology, 8(10):868-873, 2001.

[23] Wen Jiang, Zongli Li, Zhixian Zhang, Matthew L Baker, Peter E Prevelige, and Wah Chiu. Coat protein fold and maturation transition of bacteriophage P22 seen at subnanometer resolutions. Nature structural biology, 10(2):131-135, 2003.

[24] Irina I Serysheva, Steven J Ludtke, Matthew L Baker, Yao Cong, Maya Topf, David Eramian, Andrej Sali, Susan L Hamilton, and Wah Chiu. Subnanometer-resolution electron cryomicroscopybased domain models for the cytoplasmic region of skeletal muscle RyR channel. Proceedings of the National Academy of Sciences of the United States of America, 105(28):9610-9615, 2008.

[25] Preeti Gipson, Matthew L Baker, Desislava Raytcheva, Cameron Haase-Pettingell, Jacqueline Piret, Jonathan a King, and Wah Chiu. Protruding knob-like proteins violate local symmetries in an icosahedral marine virus. Nature communications, 5:4278, 2014.

[26] Matthew L Baker, Corey F Hryc, Qinfen Zhang, Weimin Wu, Joanita Jakana, Cameron HaasePettingell, Pavel V Afonine, Paul D Adams, Jonathan a King, Wen Jiang, and Wah Chiu. Validated near-atomic resolution structure of bacteriophage epsilon15 derived from cryo-EM and modeling. Proceedings of the National Academy of Sciences of the United States of America, 110(30):12301-6, 2013.

[27] Rui Zhang, Corey F Hryc, Yao Cong, Xiangan Liu, Joanita Jakana, Rodion Gorchakov, Matthew L Baker, Scott C Weaver, and Wah Chiu. 4.4 Åcryo-EM structure of an enveloped alphavirus Venezuelan equine encephalitis virus. The EMBO journal, 30(18):3854-3863, 2011.

[28] Xiangan Liu, Qinfen Zhang, Kazuyoshi Murata, Matthew L Baker, Matthew B Sullivan, Caroline Fu, Matthew T Dougherty, Michael F Schmid, Marcia S Osburne, Sallie W Chisholm, and Wah Chiu. Structural changes in a marine podovirus associated with release of its genome into Prochlorococcus. Nature structural \& molecular biology, 17(7):830-836, 2010.

[29] Steven J. Ludtke, Matthew L. Baker, Dong Hua Chen, Jiu Li Song, David T. Chuang, and Wah Chiu. De Novo Backbone Trace of GroEL from Single Particle Electron Cryomicroscopy. Structure, 16(3):441-448, 2008

[30] Junjie Zhang, Matthew L Baker, Gunnar F Schröder, Nicholai R Douglas, Stefanie Reissmann, Joanita Jakana, Matthew Dougherty, Caroline J Fu, Michael Levitt, Steven J Ludtke, Judith Frydman, and Wah Chiu. Mechanism of folding chamber closure in a group II chaperonin. Nature, 463(7279):379-383, 2010.

[31] Maofu Liao, Erhu Cao, David Julius, and Yifan Cheng. Structure of the TRPV1 ion channel determined by electron cryo-microscopy. Nature, 504(7478):107-12, 2013.

[32] Xuekui Yu, Lei Jin, and Z Hong Zhou. 3.88 A structure of cytoplasmic polyhedrosis virus by cryo-electron microscopy. Nature, 453(7193):415-419, 2008. 
[33] C. Yang, G. Ji, H. Liu, K. Zhang, G. Liu, F. Sun, P. Zhu, and L. Cheng. Cryo-EM structure of a transcribing cypovirus, 2012.

[34] Matthew L. Baker, Mariah R. Baker, Corey F. Hryc, Tao Ju, and Wah Chiu. Gorgon and pathwalking: Macromolecular modeling tools for subnanometer resolution density maps. In Biopolymers, volume 97, pages 655-668, 2012.

[35] Mariah R. Baker, Ian Rees, Steven J. Ludtke, Wah Chiu, and Matthew L. Baker. Constructing and validating initial C?? models from subnanometer resolution density maps with pathwalking. Structure, 20(3):450-463, 2012.

[36] D L Applegate, R E Bixby, V Chvátal, and W J Cook. The traveling salesman problem: a computational study. Princeton: Princeton University Press, 2006.

[37] Keld Helsgaun. General k-opt submoves for the Lin-Kernighan TSP heuristic. Mathematical Programming Computation, 1(2-3):119-163, 2009.

[38] Matthew L. Baker, Tao Ju, and Wah Chiu. Identification of Secondary Structure Elements in Intermediate-Resolution Density Maps. Structure, 15(1):7-19, 2007.

[39] Guizhen Fan, Matthew L. Baker, Zhao Wang, Mariah R. Baker, Pavel A. Sinyagovskiy, Wah Chiu, Steven J. Ludtke, and Irina I. Serysheva. Gating machinery of InsP3R channels revealed by electron cryomicroscopy. Nature, 2015.

[40] Eric F. Pettersen, Thomas D. Goddard, Conrad C. Huang, Gregory S. Couch, Daniel M. Greenblatt, Elaine C. Meng, and Thomas E. Ferrin. UCSF Chimera - A visualization system for exploratory research and analysis. Journal of Computational Chemistry, 25(13):1605-1612, 2004.

[41] M. N. Nguyen, K. P. Tan, and M. S. Madhusudhan. CLICK - Topology-independent comparison of biomolecular 3D structures. Nucleic Acids Research, 39(SUPPL. 2), 2011.

[42] Xing Zhang, Ethan Settembre, Chen Xu, Philip R Dormitzer, Richard Bellamy, Stephen C Harrison, and Nikolaus Grigorieff. Near-atomic resolution using electron cryomicroscopy and single-particle reconstruction. Proceedings of the National Academy of Sciences of the United States of America, 105(6):1867-1872, 2008

[43] Steven J. Ludtke, Matthew L. Baker, Dong Hua Chen, Jiu Li Song, David T. Chuang, and Wah Chiu. De Novo Backbone Trace of GroEL from Single Particle Electron Cryomicroscopy. Structure, 16(3):441-448, 2008

[44] K Murata, K Mitsuoka, T Hirai, T Walz, P Agre, J B Heymann, A Engel, and Y Fujiyoshi. Structural determinants of water permeation through aquaporin-1. Nature, 407(6804):599-605, 2000 .

[45] Wen Jiang, Matthew L Baker, Joanita Jakana, Peter R Weigele, Jonathan King, and Wah Chiu. Backbone structure of the infectious epsilon15 virus capsid revealed by electron cryomicroscopy. Nature, 451(7182):1130-1134, 2008.

[46] Markus Wiederstein, Markus Gruber, Karl Frank, Francisco Melo, and Manfred J. Sippl. Structurebased characterization of multiprotein complexes. Structure, 22(7):1063-1070, 2014.

[47] Maofu Liao, Erhu Cao, David Julius, and Yifan Cheng. Structure of the TRPV1 ion channel determined by electron cryo-microscopy. Nature, 504(7478):107-12, 2013.

[48] Erhu Cao, Maofu Liao, Yifan Cheng, and David Julius. TRPV1 structures in distinct conformations reveal activation mechanisms. Nature, 504(7478):113-8, 2013.

[49] Zhao Wang, Corey F. Hryc, Benjamin Bammes, Pavel V. Afonine, Joanita Jakana, Dong-Hua Chen, Xiangan Liu, Matthew L. Baker, Cheng Kao, Steven J. Ludtke, Michael F. Schmid, Paul D. Adams, and Wah Chiu. An atomic model of brome mosaic virus using direct electron detection and real-space optimization. Nature Communications, 5:4808, 2014.

[50] Alexey Amunts, Alan Brown, Xiao-chen Bai, Jose L Llácer, Tanweer Hussain, Paul Emsley, Fei Long, Garib Murshudov, Sjors H W Scheres, and V Ramakrishnan. Structure of the yeast mitochondrial large ribosomal subunit. Science (New York, N.Y.), 343(6178):1485-9, 2014.

[51] Andrew S. Doré, Nicholas Furnham, Owen R. Davies, Bancinyane L. Sibanda, Dimitri Y. Chirgadze, Stephen P. Jackson, Luca Pellegrini, and Tom L. Blundell. Structure of an Xrcc4-DNA ligase IV yeast ortholog complex reveals a novel BRCT interaction mode. DNA Repair, 5(3):362-368, 2006.

[52] Matthew L. Baker, Mariah R. Baker, Corey F. Hryc, and Frank DiMaio. Analyses of Subnanometer Resolution Cryo-EM Density Maps, volume 483. 2010.

[53] Guang Tang, Liwei Peng, Philip R. Baldwin, Deepinder S. Mann, Wen Jiang, Ian Rees, and Steven J. Ludtke. EMAN2: An extensible image processing suite for electron microscopy. Journal of Structural Biology, 157(1):38-46, 2007. 
A

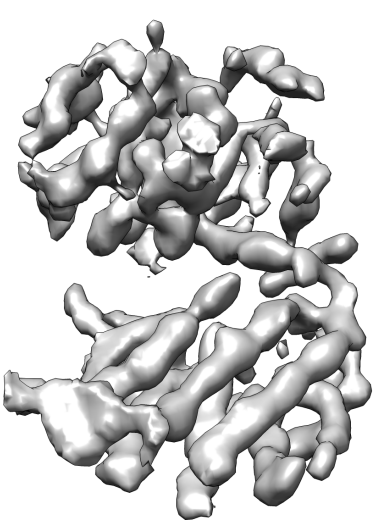

D

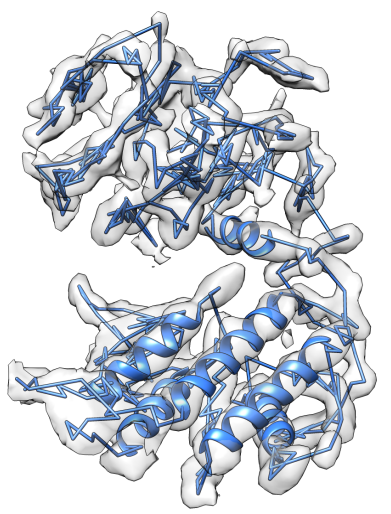

B

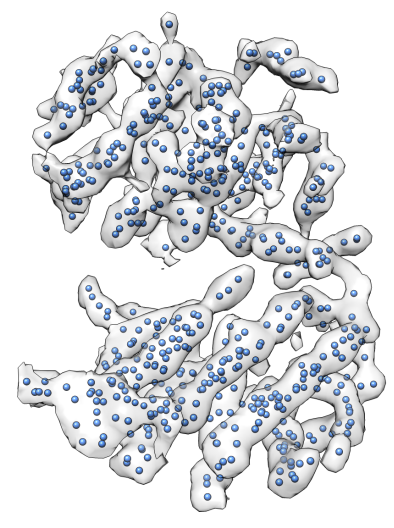

E

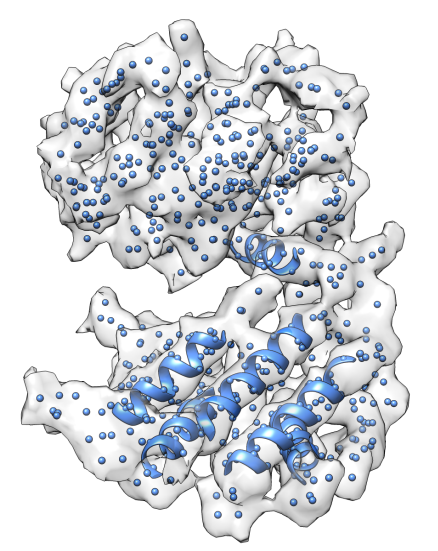

C

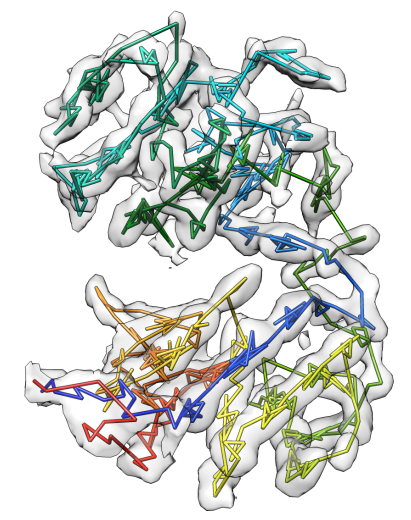

Preprocess density map (A)

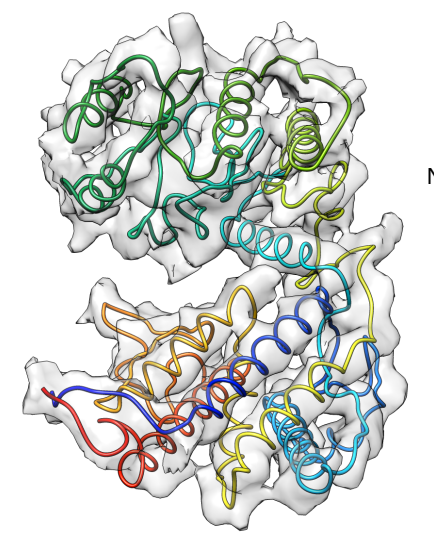

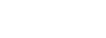

Final

modification (F)

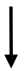

Finish

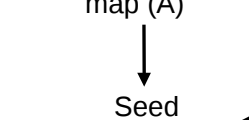

pseudoatoms (B)

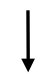

Backbone

tracing $(\mathrm{C})$<smiles>[CH][CH]</smiles>

Find and correct SSEs (D)

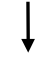

Fix SSEs in $\operatorname{map}(E)$

No new SSE

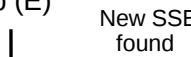

New SSE
found

Figure 1: Pathwalker work flow. (A) One subunit of the GroEL(EMDB ID: 5001, 4.2 $\AA$ resolution) is segmented out form the full GroEL density map. (B) Pseudoatoms are seeded in the density map. (C) Initial backbone tracing is performed using the pseudoatoms from B. (D) Secondary structure element are identified based on the density map and initial path. (E) The pseudoatoms are reseeded at with the fixed secondary structures. (F) Final result after multiple iterations of Pathwalking automated path modification. 


\section{Density map Pathwalking model PDB model Error by residue}

A
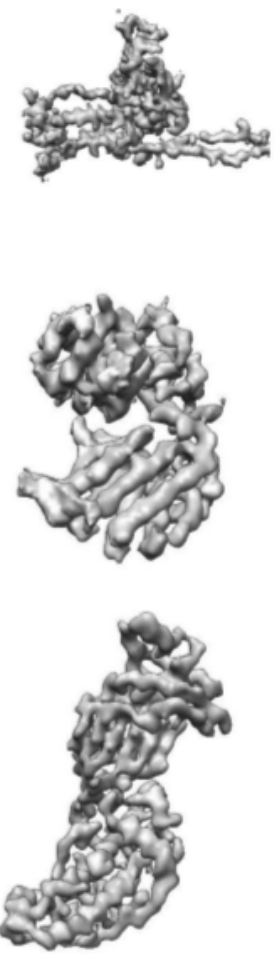

D

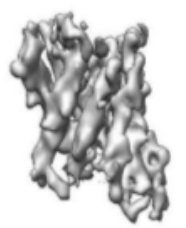

E

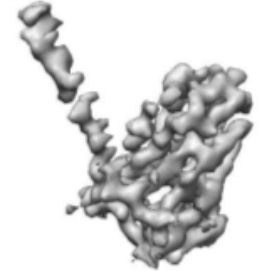

$\mathrm{F}$

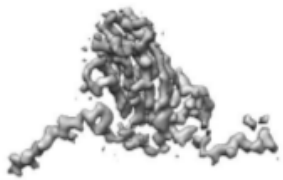

G

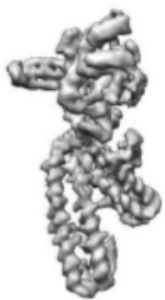

$\mathrm{H}$

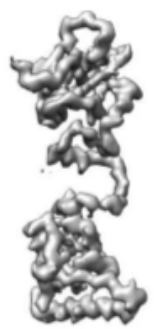

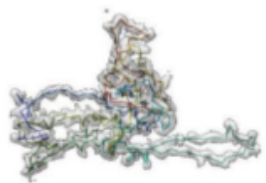
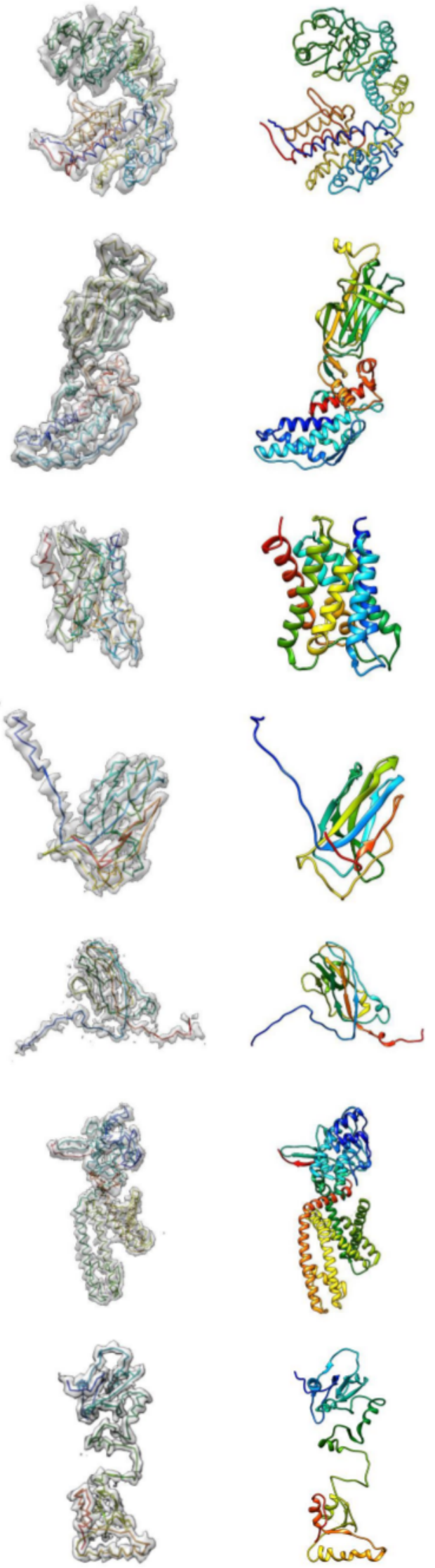
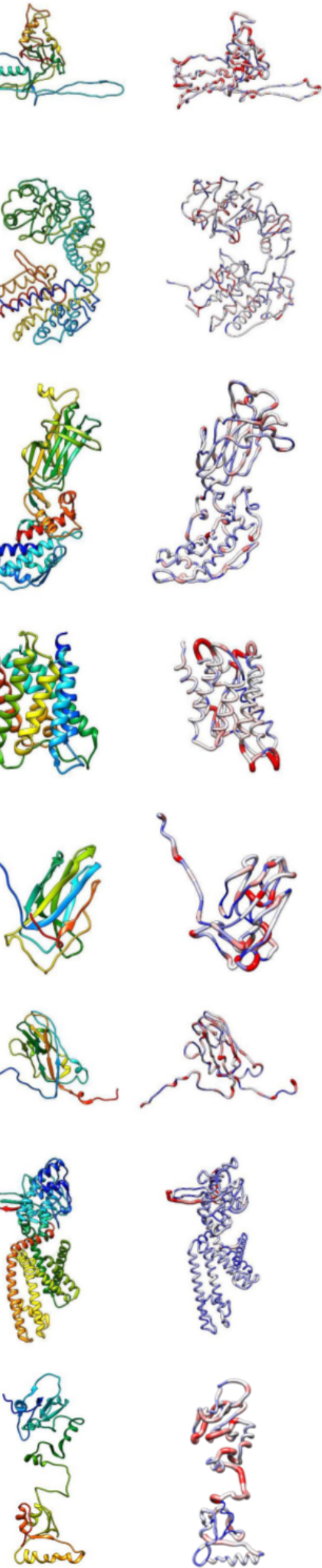

Figure 2: Pathwalking results. Each row shows an example Pathwalking result. From left to right, the columns represent the density map, final model generated by Pathwalking, corresponding X-ray model and error between the two models. (A) Epsilon15 GP7(PDB ID: 3C5B, EMDB ID: 5003). (B) GroEL chain A(PDB ID: 1SS8, EMDB ID: 5001). (C) Rotavirus, VP6(PDB ID: 1QHD, EMDB ID: 1461). (D) Aquaporin(PDB ID: 1IH5). (E) Bordetella bacteriophage, Cement protein(PDB ID: 3J4U, EMDB ID: 5764). (F) Brome Mosaic Virus, subunit A(PDB ID: 3J7L, EMDB ID: 6000). (G) TRPV1(PDB ID: 3J5P, EMDB ID: 5778).(H) DNA ligase IV chain C (PDB ID: 1Z56). 


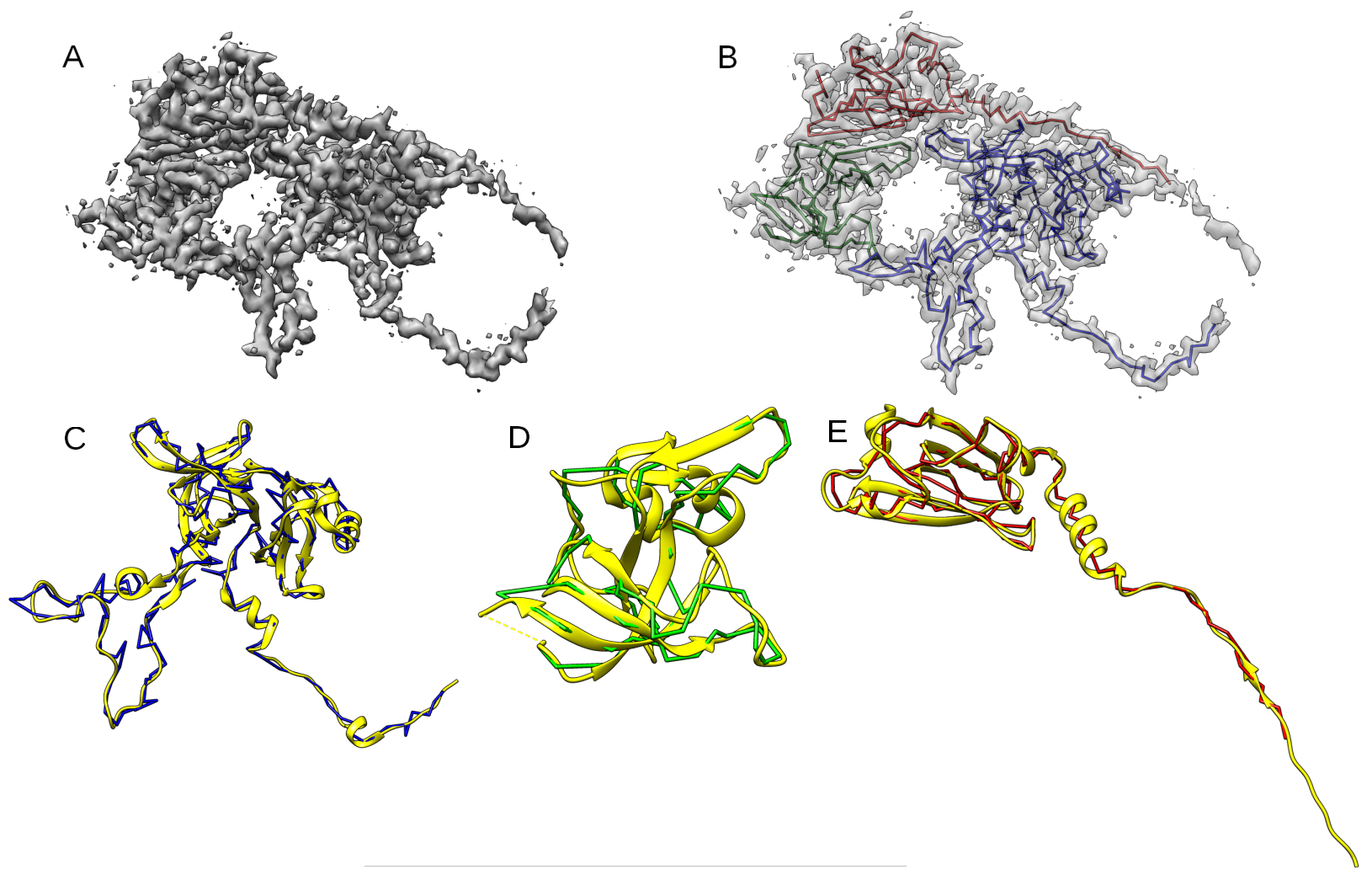

Figure 3: Multiple subunits modeling with Pathwalking. (A) Segmented density map that contains three chains of the yeast mitochondrial large ribosomal subunit(EMDB ID: 2566). (B) The resulting three subunit model from Pathwalking. Each chain is colored uniquely. (C-E) Comparison of the ground truth (yellow) are shown for each of the three chains in B. Subunit C (blue, C). Subunit I (green, D). Subunit $\mathrm{M}(\mathrm{red}, \mathrm{E})$ 
A

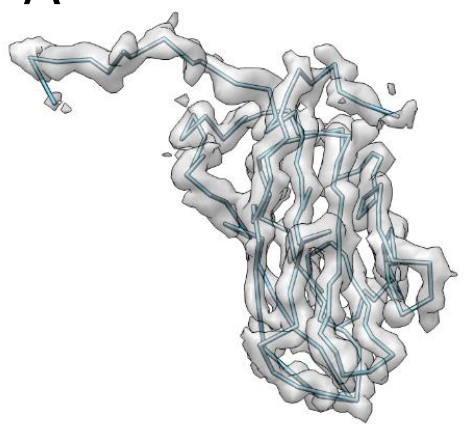

$\mathrm{D}$

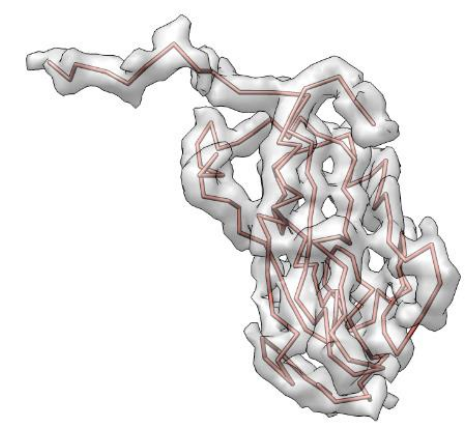

B

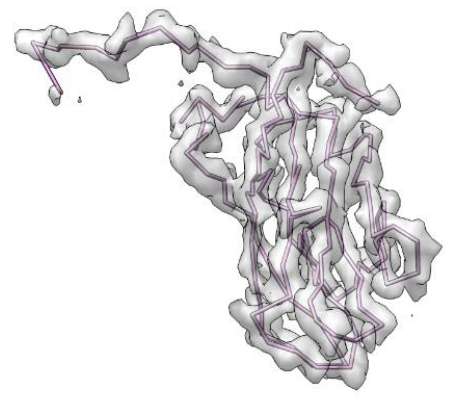

C

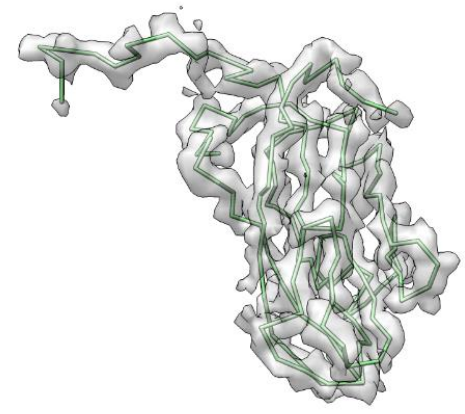

$\mathrm{E}$

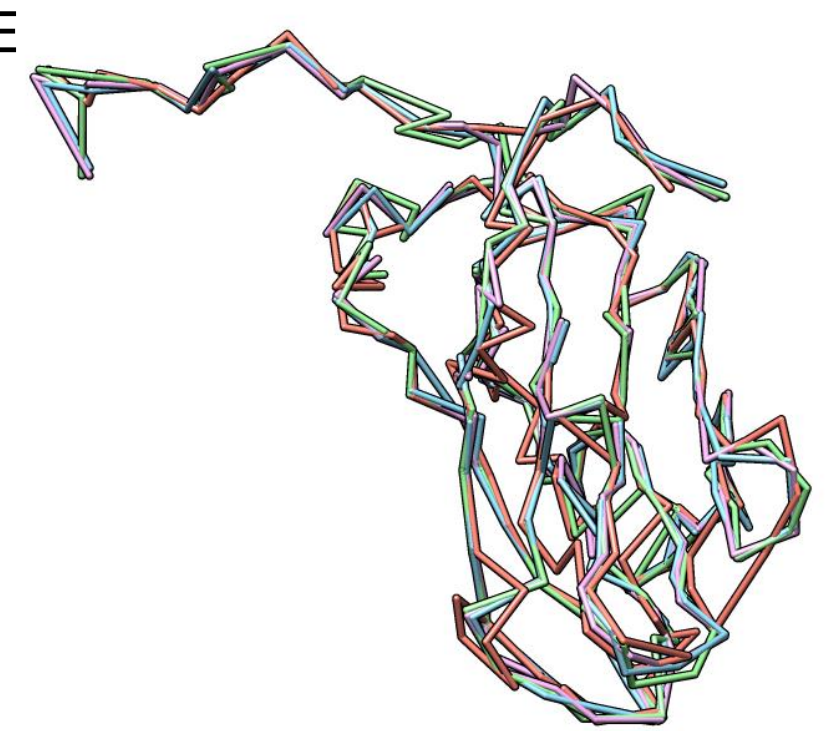

Figure 4: Pathwalking model of BMV at different resolution. (A) A capsid subunit model from a BMV reconstruction using the half dataset at $4.1 \AA$ resolution. (B) Model generated from $1 / 4$ of the dataset, $4.2 \AA$ resolution. (C) Model generated from $1 / 8$ of the dataset, $4.3 \AA$ resolution. (D) Model generated from $1 / 16$ of the dataset, $5.37 \AA$ resolution. (E) Overlap of the models shown in A-D. 

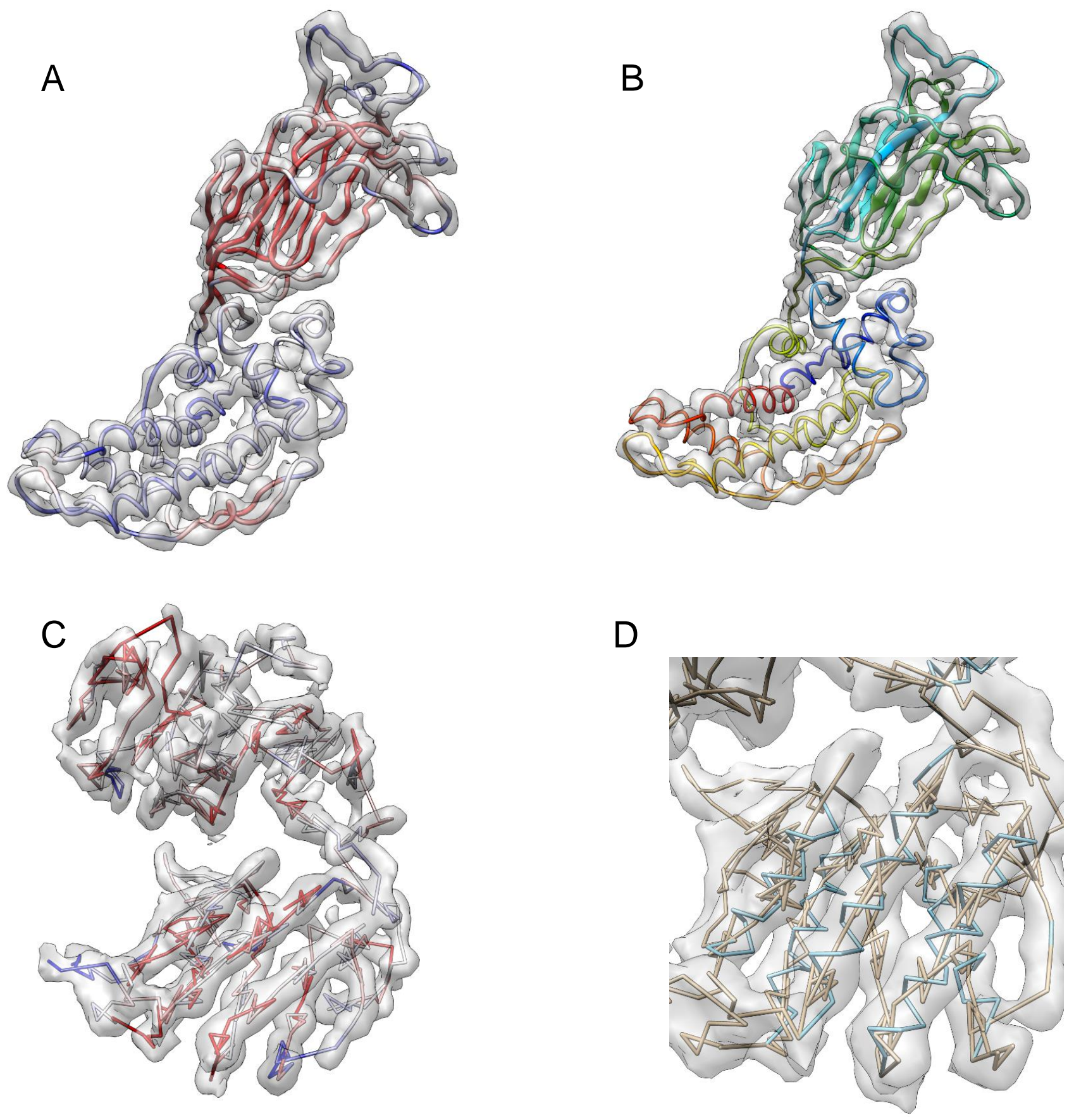

Figure 5: SSE identification protocol in Pathwalking. (A) $\beta$-sheets score. Here red color suggests possible $\beta$-sheet regions. (B) Path tracing after $\beta$-sheet identification. (C) $\alpha$-helix score. Red color suggest possible $\alpha$-helix regions. (D) Ideal helix atoms are created on the confirmed helical sites and fitted into the density. (Yellow: initial backbone trace; Blue: Corrected $\mathrm{C} \alpha$ atoms in the helices. 